\title{
Rhabdomyosarcoma Arising in a Giant Congenital Melanocytic Naevus: Case Report and Literature Review
}

\author{
Tirelo M. Pitjadia, b Reubina Wadee ${ }^{a, c}$ Wayne Grayson ${ }^{a, b}$ \\ aDivision of Anatomical Pathology, School of Pathology, Faculty of Health Sciences, \\ University of the Witwatersrand, Johannesburg, South Africa; ${ }^{b}$ Ampath National \\ Laboratories, Johannesburg, South Africa; ${ }^{c}$ National Health Laboratory Service, \\ Johannesburg, South Africa
}

\section{Keywords}

Giant congenital melanocytic naevus · Rhabdomyosarcoma · Malignant transformation · Skin

\begin{abstract}
Giant congenital melanocytic naevi (GCN) are rare, disfiguring lesions which carry a significant risk of malignant transformation. Melanoma is the most common malignancy documented in association with these lesions. Although exceedingly rare, other malignant neoplasms, including mesenchymal tumours such as rhabdomyosarcoma (RMS), may complicate GCN. This report documents a fatal embryonal RMS arising in a GCN on the distal left lower limb of a 4-month-old female infant, who had ipsilateral inguinal lymph node metastases at the time of presentation. To date there have been only 7 prior reports in the English literature of RMS complicating GCN. Differential diagnoses include small cell melanoma, rhabdoid melanoma, and melanoma with divergent RMS differentiation. A distinction between the latter and de novo RMS arising in GCN may have potential prognostic and therapeutic implications.
\end{abstract}

\section{Introduction}

Giant congenital melanocytic naevi (GCN) are defined as congenital naevi measuring more than $20 \mathrm{~cm}$ in the greatest dimension [1]. These rare lesions affect between 1 in 200,000 and 1 in 500,000 live-born infants [2]. GCN may be associated with a variety of syndromes, including Carney complex, epidermal naevus syndrome, neurofibromatosis type 1, premature

T.M.P. was employed by the National Health Laboratory Service during the initial drafting of this manuscript but is currently a full-time employee of Ampath National Laboratories in Johannesburg, South Africa. 


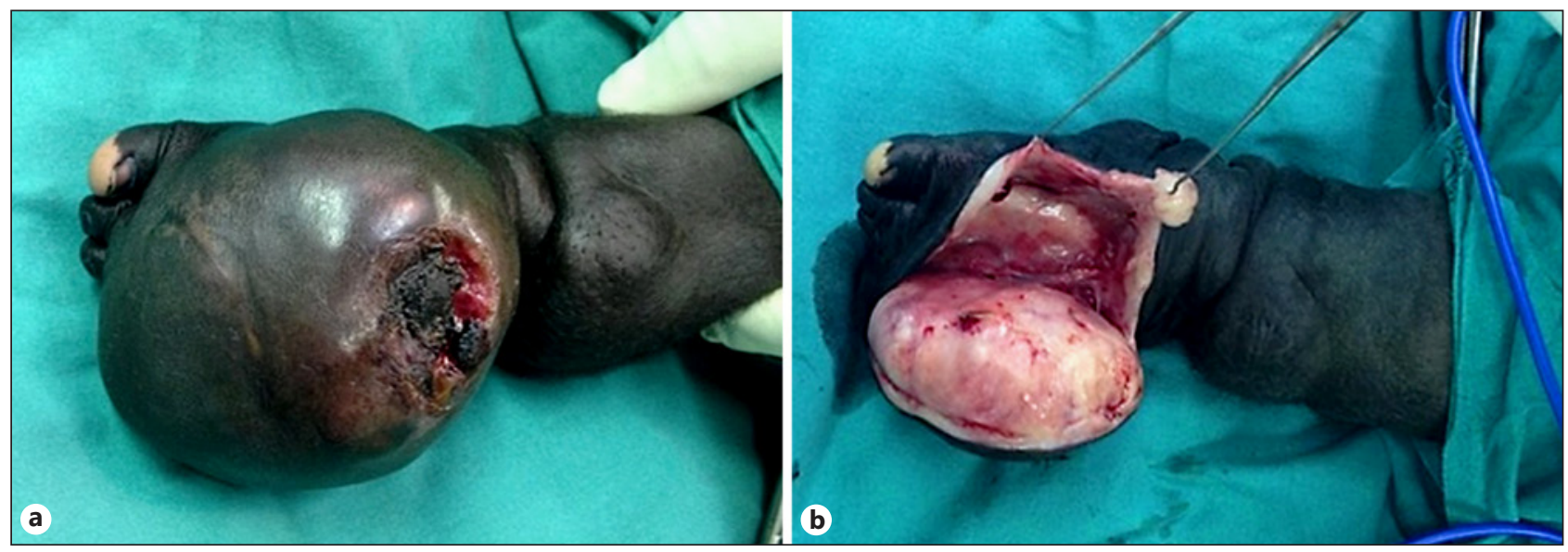

Fig. 1. a Intraoperative image of the left lower leg mass arising in a background giant congenital naevus. b Further dissection revealed a cream-coloured, relatively circumscribed tumour.

ageing syndrome, vitiligo, and neurocutaneous melanosis [1]. These pigmented, disfiguring lesions usually occur in a garment distribution (including so-called bathing trunk naevi) and may display tufts of hair on their surface [1-4]. They carry a significant risk of malignant transformation, with recorded rates ranging from 2 to $31 \%$ [1]. Melanoma represents the most common malignancy arising in the context of GCN. There are, however, rare reports of other malignant neoplasms complicating pre-existing GCN, including malignant peripheral nerve sheath tumour, liposarcoma, undifferentiated malignant spindle cell neoplasm, and rhabdomyosarcoma (RMS) [5, 6]. To date only 7 cases of transformation to RMS have been documented in the English medical literature [2-8]. Herein we report the eighth example of RMS arising in a GCN.

\section{Case Report}

The patient was a 4-month-old female infant with a GCN on the left lower leg which extended onto the foot. She presented with a firm, focally ulcerated, $10 \times 15 \mathrm{~cm}$ mass on the dorsum of the left foot within the GCN (Fig. 1a) with associated left inguinal lymphadenopathy. Intraoperatively, the mass appeared relatively circumscribed and cream coloured (Fig. 1b).

The tumour was resected with a portion of the overlying naevus. An enlarged ipsilateral inguinal lymph node was excised simultaneously and submitted for histopathological examination. The gross specimens comprised a cream-coloured, $6.5 \times 6.6 \times 3.5 \mathrm{~cm}$ tumour partially surfaced by skin, and an enlarged lymph node measuring $1.8 \mathrm{~cm}$ in maximum dimension. Microscopic examination of the upper portion of the skin excision specimen revealed part of a compound melanocytic naevus exhibiting a morphology characteristic of congenital-type naevus, with extension of naevomelanocytes around dermal nerves, blood vessels and skin adnexal structures in the reticular dermis, and permeation into the superficial subcutis (Fig. 2a, b). The naevus harboured areas of adipocytic metaplasia and exhibited prominent neurotisation in the form of Wagner-Meissner corpuscle formation (Fig. 2c). The deeper aspect of the aforementioned GCN, however, showed abrupt transition to a comparatively hypercellular, mitotically active, atypical spindle cell proliferation (Fig. 2d, 3a, b).

The individual malignant cells showed an increased nuclear-to-cytoplasmic ratio, an oval-to-elongated hyperchromatic nucleus, and one or more indistinct nucleoli. There was 

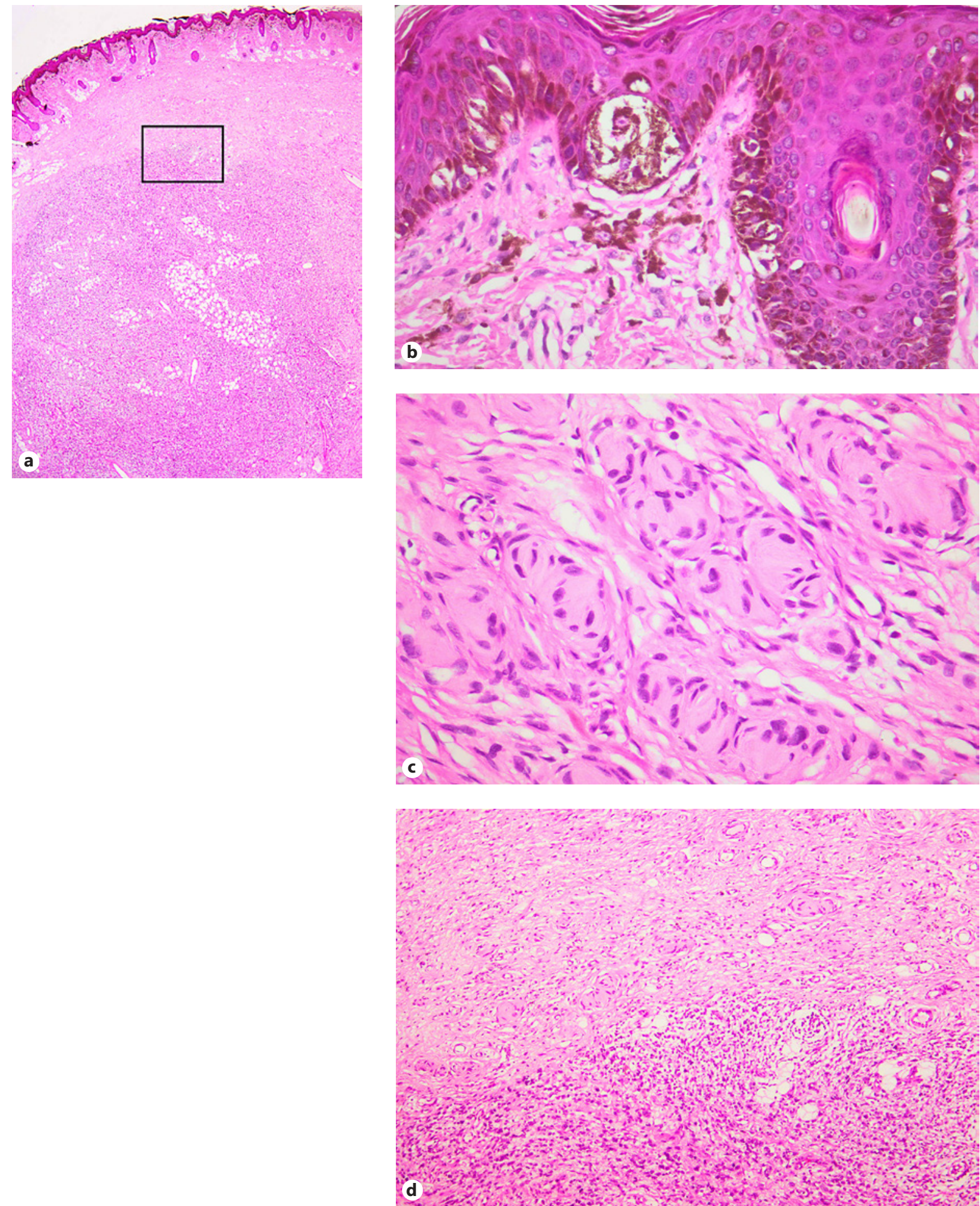

Fig. 2. Rhabdomyosarcoma arising in a giant congenital naevus. a This low-power view shows the naevus and its abrupt transition to a more cellular neoplasm, which infiltrates the subcutis. b, c The nested and focally lentiginous banal junctional component of the naevus (b) is associated with a dermal component showing striking neurotisation in the form of differentiation towards Wagner-Meissner corpuscles (c). d Detail of the area of transition, showing a banal naevus with neuroid features (upper half of field) and the contrasting malignant spindle cell neoplasm (lower half of field); the latter image corresponds to the boxed area in a. 


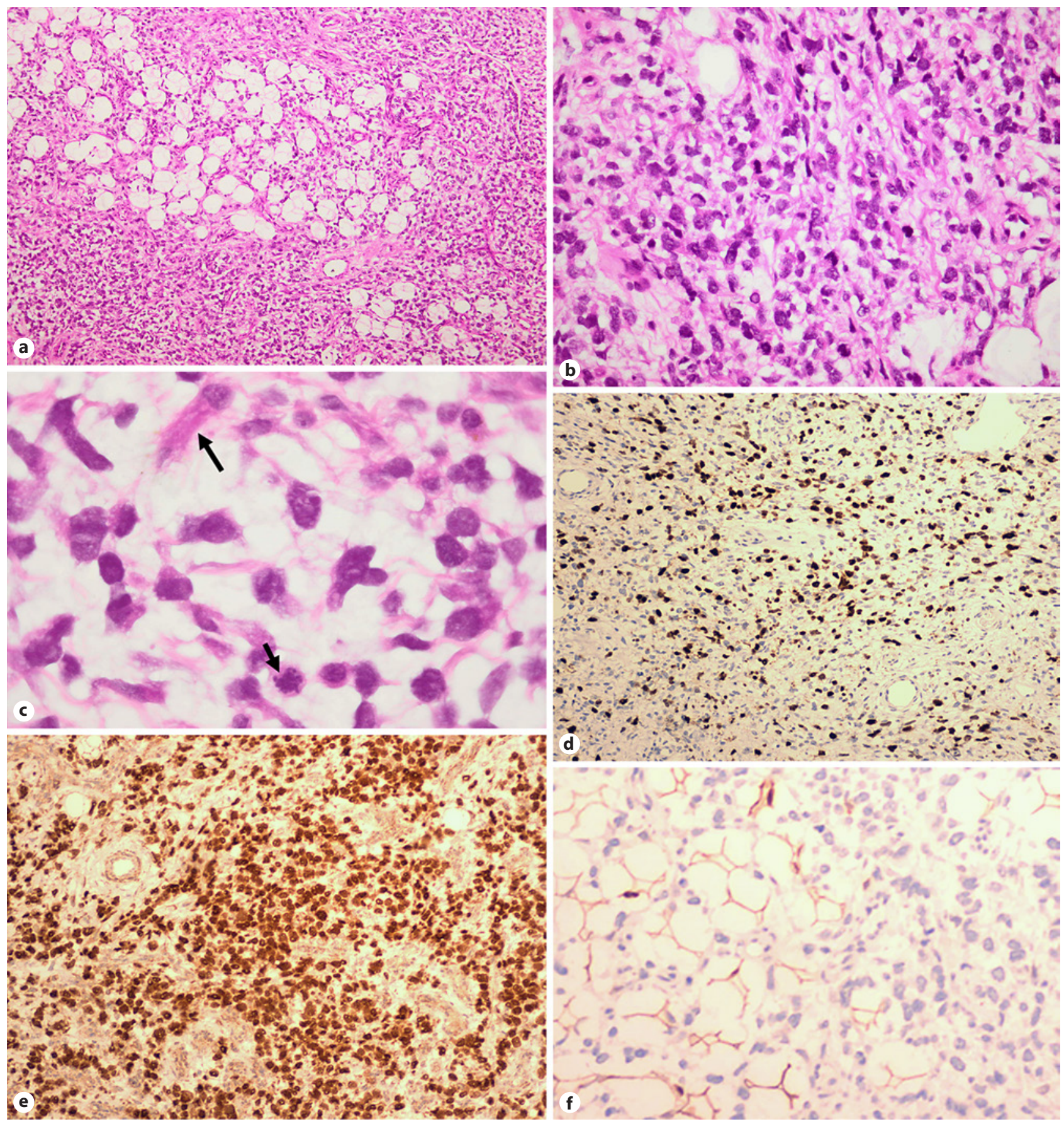

Fig. 3. Embryonal rhabdomyosarcomatous component. The hypercellular malignant neoplasm infiltrating the subcutis $(\mathbf{a}, \mathbf{b})$ is composed of primitive plump-to-spindled cells (c) showing mitotic activity (short arrow) and a rhabdomyoblastic morphology, including occasional characteristic "tadpole-like" forms (long arrow). Nuclear immunoreactivity for myogenin (d) and MyoD1 (e) confirms the presence of neoplastic skeletal muscle differentiation. The absence of immunostaining with melanocytic markers such as S100 protein (f) rules out a melanoma. 

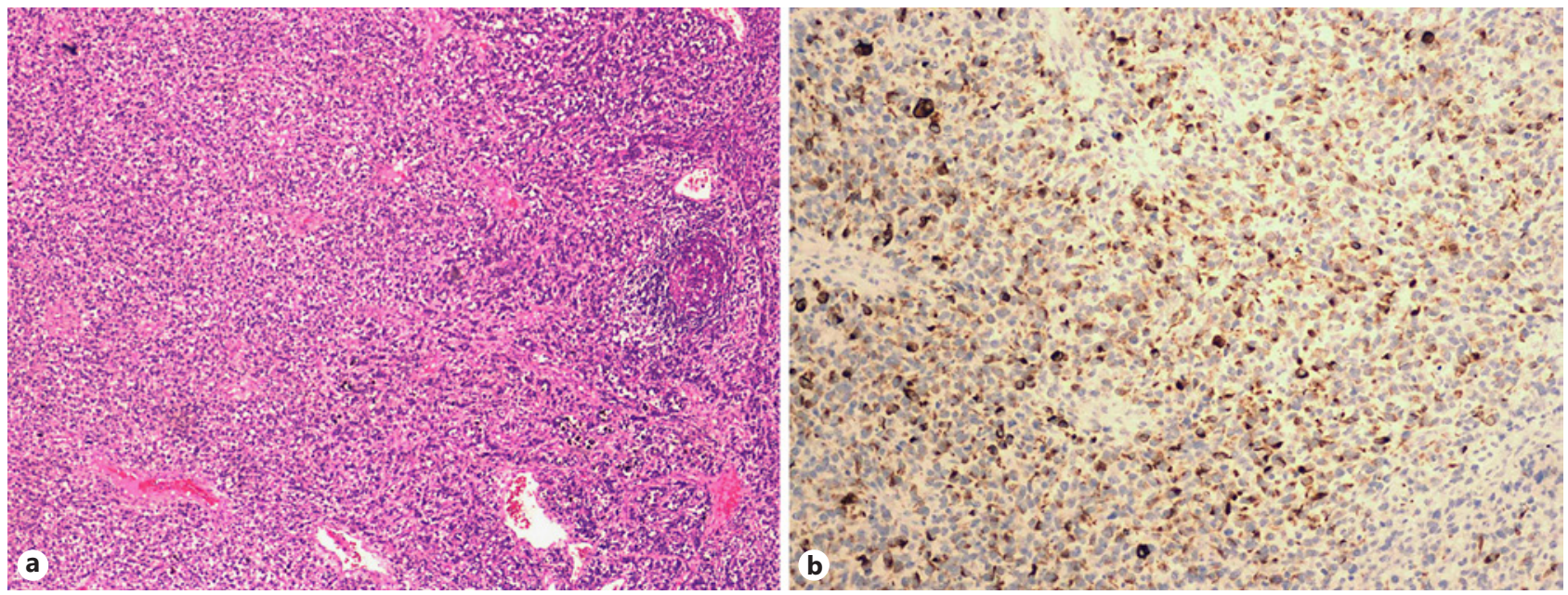

Fig. 4. Medium-power photomicrograph of the resected inguinal lymph node (a) showing partial effacement by sheets of atypical small round cells (left) and adjacent residual nodal parenchyma (right). The intranodal neoplastic cellular infiltrate exhibits diffuse immunoreactivity for desmin (b), confirming the presence of metastatic rhabdomyosarcoma.

eccentric nuclear placement in some cells, with adjacent elongated eosinophilic cytoplasm and a resultant "tadpole-like" appearance (Fig. 3c). Definitive intracytoplasmic cross-striations, however, were not evident. The malignant cells demonstrated positive immunoreactivity for desmin, MyoD1, and myogenin, confirming the presence of skeletal muscle differentiation (Fig. 3d, e), while immunohistochemical (IHC) markers of melanocytic differentiation, namely, S100 protein (Fig. 3f), melan-A (MART1), HMB-45, and microphthalmia-associated transcription factor (MITF) proved negative.

The resected lymph node was partially effaced by a morphologically and immunophenotypically identical malignant cellular population, diagnostic of metastatic RMS (Fig. 4). Neither the main excision specimen nor the involved lymph node showed evidence of a melanoma component. The patient was referred to a paediatric oncologist for further management, including appropriate chemotherapeutic intervention. She did not, however, attend her scheduled follow-up appointment. It was later gleaned from the infant's mother that she had died 3 months after the diagnosis had been made.

\section{Discussion}

Congenital melanocytic naevi are relatively common benign lesions which carry a potential risk for transformation to melanoma. They are found in approximately $1 \%$ of newborn infants [1]. When exceeding $20 \mathrm{~cm}$ in their greatest dimension, they are designated GCN. These lesions typically present as irregular, disfiguring, hyperpigmented lesions on the trunk in a "bathing trunk" distribution and may show hair on their surface. They are usually present at birth or develop soon thereafter [1]. Histologically, GCN may be junctional, intradermal, or compound. Involvement of eccrine ducts and connective tissue septa by the naevus cells is regarded as more specific for congenital naevi [1]. Other features that have been proposed as being more indicative of congenital naevi include single-cell or "Indian file-like" infiltration of dermal collagen bundles and extension of naevus cells around dermal vessels and nerves. 
Table 1. Synopsis of reported cases of RMS arising in congenital melanocytic naevi

\begin{tabular}{|c|c|c|c|c|c|}
\hline Study [Ref.] & Age & Gender & Anatomical site & Histological type & Outcome \\
\hline Chikhalkar et al. [2] & 21 years & M & $\begin{array}{l}\text { Rt lower abdominal wall } \\
\text { and iliac fossa }\end{array}$ & Alveolar RMS & DOD within 1 month \\
\hline Hoang et al. [3] & 4 years & $\mathrm{F}$ & Lt gluteal and sacral area & Embryonal RMS & DOD at 5 years of age \\
\hline Ilyas et al. [4] & 6 weeks & $\mathrm{F}$ & $\begin{array}{l}\text { Lower back, extending } \\
\text { onto abdomen }\end{array}$ & Embryonal RMS & $\begin{array}{l}\text { ANED at } 6 \text { months of } \\
\text { follow-up }\end{array}$ \\
\hline Hendrickson and Ross [5] & 7 months & $\mathrm{F}$ & $\begin{array}{l}\text { Lt side of scalp and } \\
\text { Lt cheek }\end{array}$ & $\begin{array}{l}\text { Lipoblastic and } \\
\text { rhabdomyoblastic } \\
\text { differentiation }\end{array}$ & $\begin{array}{l}\text { Local recurrence at } 3 \\
\text { months of follow-up; } \\
\text { tumour re-excised; } \\
\text { ANED at } 3 \text { years of } \\
\text { age }\end{array}$ \\
\hline Zúñiga et al. [6] & $\begin{array}{l}\text { Newborn } \\
\text { infant }\end{array}$ & M & $\begin{array}{l}\text { Posterior neck } \\
\text { and upper trunk }\end{array}$ & Embryonal RMS & $\begin{array}{l}\text { DOD at } 11 \text { months of } \\
\text { age }\end{array}$ \\
\hline Schmitt et al. [7] & 7 months & M & Trunk and thigh & Pleomorphic RMS & $\begin{array}{l}\text { ANED at } 18 \text { months of } \\
\text { follow-up }\end{array}$ \\
\hline Christman et al. [8] & 4 months & $\mathrm{F}$ & $\begin{array}{l}\text { Apex of gluteal cleft } \\
\text { (sacral region) }\end{array}$ & Embryonal RMS & $\begin{array}{l}\text { ANED at } 6 \text { years of } \\
\text { follow-up }\end{array}$ \\
\hline Present study & 4 months & $\mathrm{F}$ & $\begin{array}{l}\text { Lt foot; Lt inguinal } \\
\text { lymph node metastasis } \\
\text { at diagnosis }\end{array}$ & Embryonal RMS & $\begin{array}{l}\text { DOD } 3 \text { months after } \\
\text { diagnosis }\end{array}$ \\
\hline
\end{tabular}

Rt, right; Lt, left; RMS, rhabdomyosarcoma; DOD, died of disease; ANED, alive with no evidence of disease.

Transformation of congenital melanocytic naevi into RMS has been documented on 7 previous occasions (Table 1) [2-8]. Six of these single-case reports were on infants or children ( 2 male, 4 female). One of the neoplasms occurred in a newborn infant, while the other paediatric patients were aged from 6 weeks to 4 years at the time of presentation. Despite the overall rarity thereof, this phenomenon is not entirely unexpected given the neural crest origin and occurrence of RMS in the paediatric age group in general. Four of the 6 previously reported paediatric cases alluded to above harboured an RMS with an embryonal morphology, similar to that seen in the present case report. Only 1 case showed pleomorphic RMS, an unusual variant in this age group. The exact histologic RMS subtype/variant in the remaining paediatric case was not specified [5].

The single recorded example of alveolar RMS arising in a GCN occurred on the right lower anterior abdominal wall of a young male adult aged 21 years [2]. Although RMS is uncommon in adults, the alveolar variant has a greater propensity to occur in the aforementioned age group [9]. Given that GCN are derived from neural crest cells, the rare evolution of malignant neoplastic mesenchymal proliferations such as RMS, malignant peripheral nerve sheath tumour, or even liposarcoma in GCN can probably be explained on the basis of the persistence of pluripotent cells of neural crest origin [3-6].

Melanoma is an important differential diagnosis to consider in this context, including small cell melanoma, rhabdoid melanoma, and melanoma with divergent rhabdomyosarcomatous differentiation. Accurate distinction is readily facilitated by judicious use of relevant IHC stains. A panel inclusive of markers such as S100, HMB-45, melan-A, MITF, and SOX10 will stain the majority of melanomas, while antibodies to desmin, MyoD1, and myogenin will confirm the presence of RMS. It is, however, important to remain cognizant of the possibility 
that some cases of rhabdoid melanoma may stain negatively with all melanoma markers. Despite their potential RMS-like morphology, such tumours should not be presumed to represent RMS without the use of confirmatory IHC stains to support definitive skeletal muscle differentiation [1]. Conversely, rare examples of RMS may exhibit a true rhabdoid phenotype [10].

The tumour most likely to pose diagnostic challenges with respect to distinction of RMS arising de novo in GCN is a melanoma with true rhabdomyosarcomatous differentiation complicating a GCN. The tumour cells in these exceptionally rare melanomas with divergent differentiation, however, show ultrastructural features and an IHC profile in support of both malignant melanocytic and skeletal muscle differentiation [11]. Other differential diagnoses that might be considered given the young age of the patients, the histomorphology, and potential cutaneous involvement include the Ewing family of tumours (e.g., peripheral neuroectodermal tumour) and neuroblastoma. Although small cell melanoma could perhaps be mistaken for one of the aforementioned small round cell tumours of childhood, occurrence of the latter in association with a cutaneous GCN is, to the best of our knowledge, hitherto unreported. Members of the Ewing family of tumours can be distinguished from RMS by the presence of IHC staining for neuron-specific enolase, pancytokeratin (AE1/3), CD99, and FLI-1 in the former, and the associated absence of staining with the skeletal muscle markers alluded to above.

In summary, this report documents the eighth reported example of RMS arising in a GCN. The fatal outcome in our case attests to the metastatic potential of these tumours. Since melanoma represents the most frequent malignant neoplasm occurring in association with GCN, thorough sampling and the judicious application of IHC stains are warranted to facilitate the distinction between melanoma and de novo RMS complicating pre-existing GCN. Since chemotherapeutic regimens exist for RMS, a distinction from melanoma in this context may have prognostic relevance.

\section{Acknowledgements}

Dr. Aimee Jenkin, former member of the Division of Plastic and Reconstructive Surgery, University of the Witwatersrand, Johannesburg, and the Charlotte Maxeke-Johannesburg Academic Hospital, Johannesburg, South Africa, is acknowledged for the intraoperative clinical images.

\section{Statement of Ethics}

Telephonic consent was obtained from the patient's mother for the use of the clinical photographs in this publication. An ethics clearance certificate was also sought from the Human Research Ethics Committee, University of the Witwatersrand, Johannesburg.

\section{Disclosure Statement}

The authors have no conflicts of interest to declare. 


\section{Author Contributions}

All three authors contributed equally to the compilation of this case report.

\section{References}

1 Weedon D. Weedon's skin pathology. 3rd ed. London: Churchill Livingstone Elsevier; 2010. p. 725-7.

2 Chikhalkar S, Gutte R, Holmukhe S, Khopkar U, Desai S, Gupta S. Alveolar rhabdomyosarcoma arising in a giant congenital melanocytic nevus in an adult - case report with review of literature. Int J Dermatol. 2013 Nov; 52(11):1372-5.

3 Hoang MP, Sinkre P, Albores-Saavedra J. Rhabdomyosarcoma arising in a congenital melanocytic nevus. Am J Dermatopathol. 2002 Feb;24(1):26-9.

4 Ilyas EN, Goldsmith K, Lintner R, Manders SM. Rhabdomyosarcoma arising in a giant congenital melanocytic nevus. Cutis. 2004 Jan; 73(1):39-43.

5 Hendrickson MR, Ross JC. Neoplasms arising in congenital giant nevi: morphologic study of seven cases and a review of the literature. Am J Surg Pathol. 1981 Mar;5(2):109-35.

6 Zúñiga S, Las Heras J, Benveniste S. Rhabdomyosarcoma arising in a congenital giant nevus associated with neurocutaneous melanosis in a neonate. J Pediatr Surg. 1987 Nov;22(11):1036-8.

7 Schmitt FC, Bittencourt A, Mendonca N, Dorea M. Rhabdomyosarcoma in a congenital pigmented nevus. Pediatr Pathol. 1992 Jan-Feb;12(1):93-8.

8 Christman MP, Kerner JK, Cheng C, Piris A, Nepo AG, Sepehr A, et al. Rhabdomyosarcoma arising in a giant congenital melanocytic nevus. Pediatr Dermatol. 2014 Sep-Oct;31(5):584-7.

9 Weiss SW, Goldblum JR. Enzinger and Weiss's soft tissue tumours. 5th ed. Philadelphia: Mosby Elsevier; 2008. p. 595-627.

10 Kodet R, Newton WA Jr, Hamoudi AB, Asmar L. Rhabdomyosarcomas with intermediate-filament inclusions and features of rhabdoid tumors. Light microscopic and immunohistochemical study. Am J Surg Pathol. 1991 Mar;15(3):257-67.

11 Antonov NK, Niedt GW. Malignant melanoma with rhabdomyosarcomatous differentiation: a case report. Am J Dermatopathol. 2016 Jun;38(6):456-60. 